\title{
Three-Axis Platform Simulation: Bond Graph and Lagrangian Approach
}

by M. J. L. TIERNEGO and J. J. VAN DIXHOORN

Twente University of Technology, Department of Electrical Engineering, 7500 AE ENSCHEDE, The Netherlands

ABSTRACT: A bond graph model is derived for the geometric constraints of a three-axis flight table. Gimbal dynamics are easily added even in asymmetrical and unbalanced cases. A method is introduced to make the local dependent inertias computable. The bond graph compares favourably to the Lagrangian approach as to modelling effort and accessibility of intermediate variables as well as having computational advantages.

\section{Nomenclature}

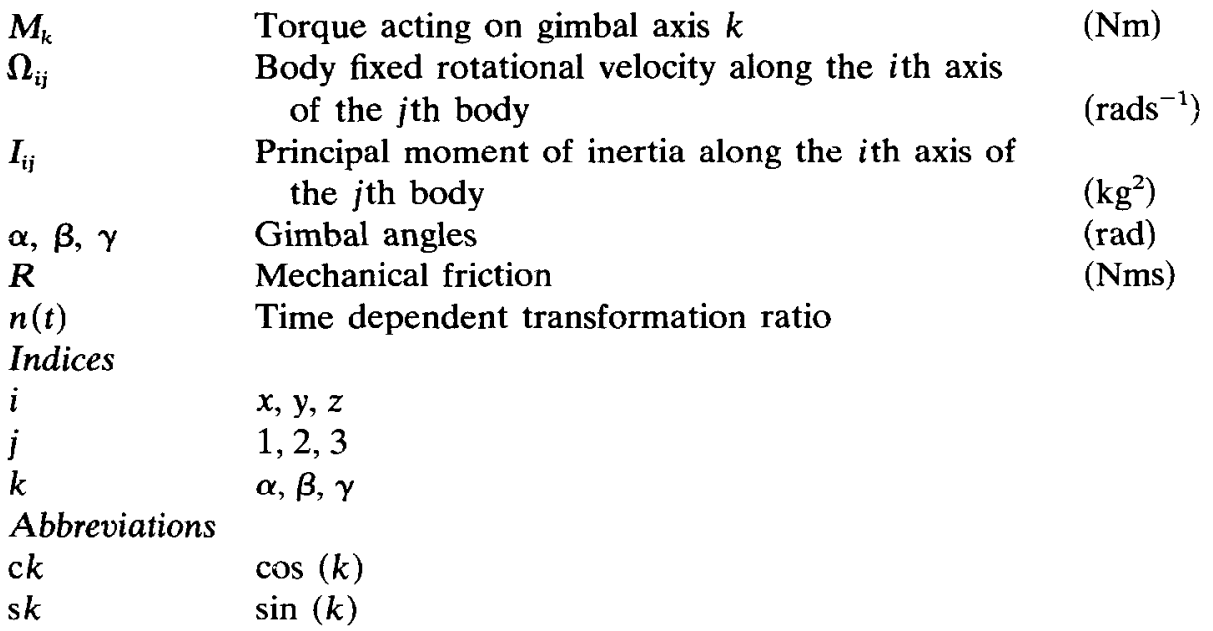

\section{Introduction}

This paper compares the bond graph technique to the Lagrangian approach in the study of three-axis platform dynamics. Both the modelling process and computational aspects will be considered. The conventional approach to the modelling of three-axis platforms is to use Lagrangian equations (7). To bring the resulting equations to a form suitable for computation, the inversion of a matrix with variable elements is necessary, which can be done either analytically or numerically at every time step. Very often, however, the equations are simplified by neglecting the cross and squared products of the velocities. This can only be justified if they exert relatively small torques. The resulting equations can be easily handled by most simulation languages. One drawback 
to the procedure, however, is that in the derivation of these equations many physical variables and components are absorbed or transformed beyond recognition.

The bond graph technique has developed into a powerful alternative tool for modelling multibody dynamic systems (6). In a bond graph the physical components and variables are represented with the topological structure relating them. In the resulting simulation model all torques and velocities are explicitly available. This has the advantage that the model can be extended to include other components, without the obligation to derive new equations. The new components will generally produce changes in the order of computation or in the arrangement of the equations. These changes can be detected from the bond graph topology itself by the use of causal analysis.

In some systems the Lagrangian approach may be the most convenient one for representing a particular part of the system while other parts could better be modelled by bond graphs. In such cases the overall representation and organization of the equations can be clearly shown in a bond graph, which includes Lagrangian submodels (8).

\section{Physical Model and Coordinate Systems}

The three-axis table consists of three symmetric and balanced gimbals, rotating about their centres of mass (Fig. 1). The angular position of the inner gimbal or table is to be controlled by individually positioning the three axes. To that end the rotation angle of each axis is measured by a pancake resolver and driven by a direct drive permanent magnet motor. Because of the inertias of the three gimbals, the three position control systems exert disturbing torques on each other. The model under consideration is used in the design of a control system in which the three controls are decoupled.

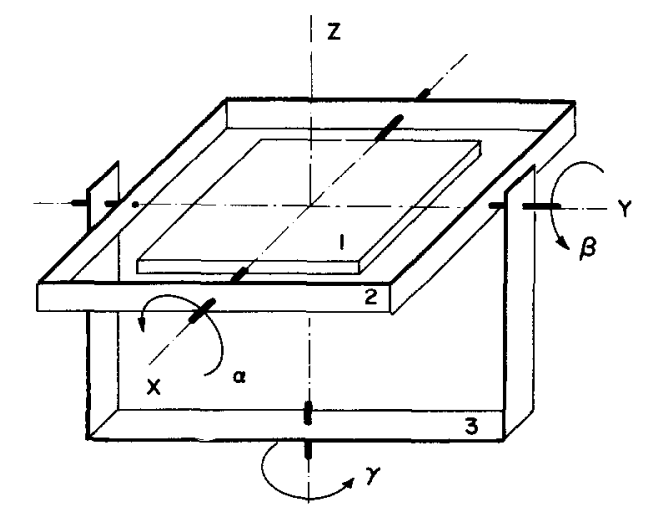

Fig. 1. Physical model, coordinate system, inertias:

inner gimbal (1)

$I_{\lambda 1}=2 \times 10^{-3} \mathrm{~kg} \mathrm{~m}^{2}$

$I_{\mathrm{y} 1}=8 \times 10^{-3} \mathrm{~kg} \mathrm{~m}^{2}$

$I_{z 1}=6 \times 10^{-3} \mathrm{~kg} \mathrm{~m}^{2}$ middle gimbal (2)

$I_{x 2}=12 \times 10^{-3} \mathrm{~kg} \mathrm{~m}^{2}$

$I_{\mathrm{y} 2}=16 \times 10^{-3} \mathrm{~kg} \mathrm{~m}^{2}$

$I_{z 2}=14 \times 10^{-3} \mathrm{~kg} \mathrm{~m}^{2}$ outer gimbal (3)

$I_{z 3}=20 \times 10^{-3} \mathrm{~kg} \mathrm{~m}^{2}$. 
For each gimbal a body coordinate system is defined coinciding with its main body axes. Coordinates are indicated by index $i$ for gimbal $i ; i=1,2,3$. The inertial reference coordinate system, to which the drive of gimbal 3 is connected, has index 0 . The angular velocities $\Omega\left(\mathrm{rads}^{-1}\right)$ of each gimbal are expressed along the positive $x, y, z$ directions of their respective body coordinate systems. So the angular velocity of gimbal 2 in the positive $z$-direction of its own $z$-axis should be called $\Omega_{2, z 2}$.

The first index 2 refers to the gimbal, the second index 2 refers to the coordinate system. Because in body coordinate system 2 , generally no other velocities will be expressed other than those of gimbal 2 , the above velocity is often written $\Omega_{z 2}$. The rotation angles of the three driven gimbal axes are called $\alpha, \beta$ and $\gamma$. The reference position, in which $\alpha=\beta=\gamma=0$, corresponds with the coincidence of all four coordinate systems, i.e. the situation represented in Fig. 1.

The angular velocities of the driven axis are called $\dot{\alpha}, \dot{\beta}$ and $\dot{\gamma}$. The angular velocities of the reference system are assumed to be zero, which implies that

$$
\begin{aligned}
& \dot{\gamma} \equiv \Omega_{\mathrm{z} 3}, \\
& \dot{\beta} \equiv \Omega_{\mathrm{y} 2} \\
& \dot{\alpha} \equiv \Omega_{\mathrm{x} 1}-\Omega_{x 2} .
\end{aligned}
$$

The last indentity is clear if it is remembered that coordinate systems 1 and 2 have the $x$-axis in common, so $\Omega_{2, x 1}=\Omega_{2, x 2}$,

$$
\dot{\alpha} \equiv \Omega_{1, x 1}-\Omega_{2, x 1}=\Omega_{1, x 1}-\Omega_{2, x 2}=\Omega_{x 1}-\Omega_{x 2} .
$$

The basic structure of a part of the model is shown in a word bond graph in Fig. 2. Gimbals 1 and 2 are nearly completely connected, but a coordinate transformation is required in between to take care of the different values of the velocities in the two coordinate systems. Also shown is the external drive in the $x$-direction which creates a velocity difference, represented by a 0 -junction.

\section{Coordinate transformations}

Coordinate systems 1, 2, 3 and 0 have, pairwise, one coordinate in common. This largely simplifies the coordinate transformation between two adjacent pairs.

In Fig. 3 the transformation of $(y, z)$ coordinates, expressed in body coordinate system 2 , to those in body coordinate system 1 is indicated. The transformation matrix $\mathbf{T}(\alpha)$ is a function of one rotation angle only. This transformation matrix applies to the angular velocities $\Omega$, which are described by vectors in these coordinate systems.

In bond graph notation the transformation is represented by the modulated multiport transformer (MTF) of Fig. 3(d). In the $x$-direction the coordinate transformation is $1: 1$, because the $x 1$ and $x 2$ axes are coincident, so

$$
\begin{aligned}
& \Omega_{1, x 1}=\Omega_{1, x 2}, \\
& \Omega_{2, x 1}=\Omega_{2, x 2} .
\end{aligned}
$$




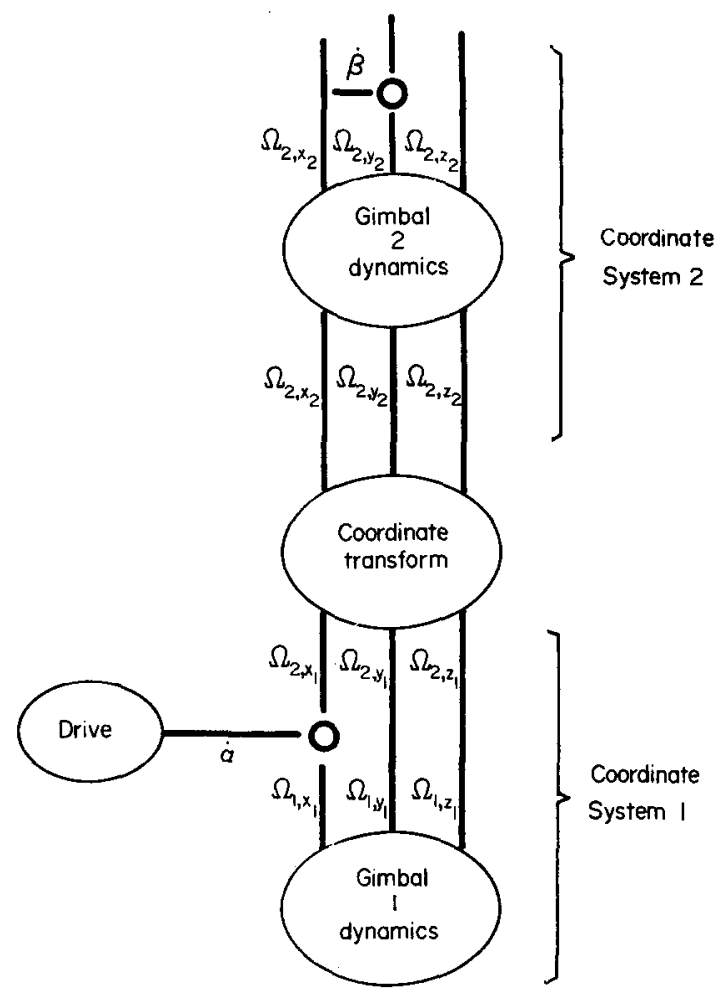

FIG. 2. General bond graph set-up.

The resulting complete structure of the geometric relationships between the angular velocities is shown for the general case in Fig. 4(a). In the specific case, in which the reference velocities $\Omega_{0}$ are zero, the structure simplifies to that of Fig. 4(b). A complete dynamic model can now easily be obtained by adding the external influences (drives) and the dynamic properties of the three gimbals. This is symbolically indicated in Fig. 5.

\section{Gimbal Dynamics}

The dynamics of each gimbal are represented by the triangular bond graph structure of Fig. 6(a) (1). $I_{x x} \ldots I_{z z}$ are the principal moments of inertia of the gimbal along the main body axes, which were chosen as body coordinate systems. The modulated gyrators MGY express the property that a velocity at one side, say $\Omega_{y}$, exerts a torque $M$ at the other side, say at $\Omega_{z}$. They are modulated gyrators, MGY, because the gyration ratio, i.e. the gyrational coupling between $\Omega_{y}$ and $\Omega_{z}$, is dependent on an external variable, i.e. $I_{x x} \cdot \Omega_{x}$. So the mentioned torque $M=I_{x x} \cdot \Omega_{x} \cdot \Omega_{y}$. The bond graph shows, that at each 

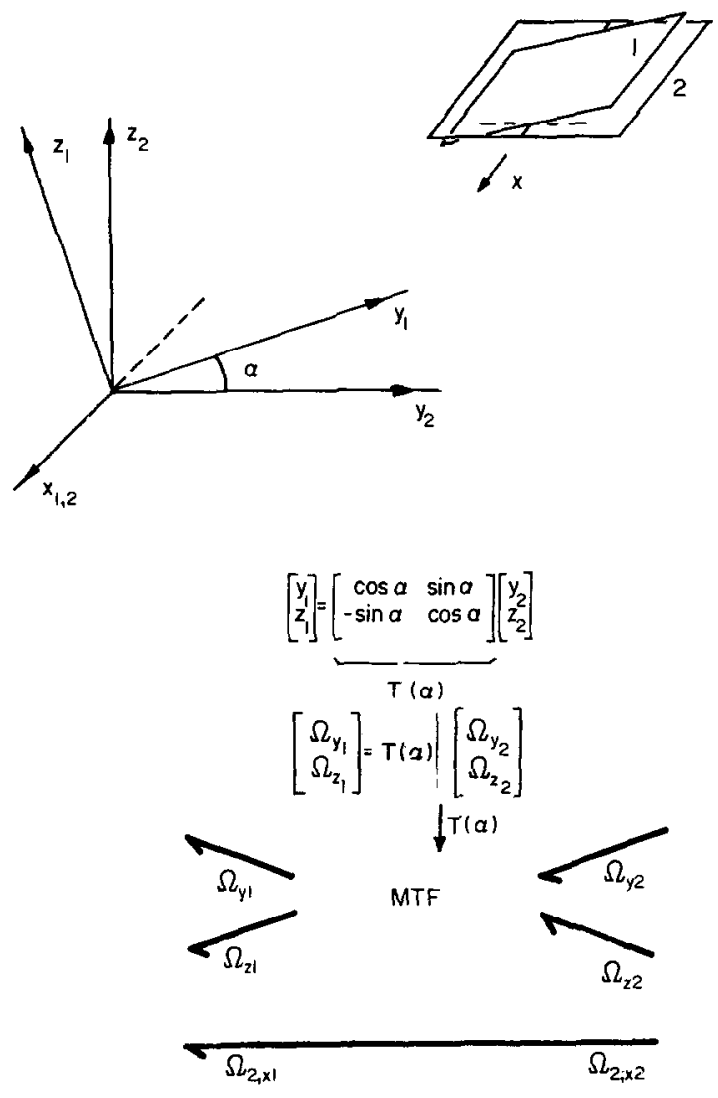

(b)

FIG. 3. MTF of planar rotation.

angular velocity or 1-junction, a summation of torques holds

$$
\begin{aligned}
& I_{z z} \cdot \dot{\Omega}_{z}=M_{z}+I_{x x} \cdot \Omega_{x} \cdot \Omega_{y}-I_{y y} \cdot \Omega_{x} \cdot \Omega_{y}, \\
& I_{x x} \cdot \dot{\Omega}_{x}=M_{x}+\left(I_{y y}-I_{z z}\right) \Omega_{y} \cdot \Omega_{z}, \\
& I_{y y} \cdot \dot{\Omega}_{y}=M_{y}+\left(I_{z z}-I_{x x}\right) \Omega_{x} \cdot \Omega_{z} .
\end{aligned}
$$

(a)

These are the well known Euler equations. They express the fact that the rate of change of the rotational momentum vector, expressed in body coordinates, $I_{z z} \cdot \dot{\Omega}_{z}$, is partly caused by the external torque $M_{z}$ but is also caused by the rotation of the body frame.

For a partly symmetrical body, in which e.g. $I_{y y}=I_{z z} \neq I_{x x}$, the bond graph reduces to the simple structure of Fig. 6(b).

\section{Complete Bond Graph Model}

In order to complete the bond graph model the drives have to be taken in account. Each drive consists of a permanent magnet dc-motor, having armature resistance $\left(R_{a}\right)$ and inductance $\left(L_{a}\right)$, as well as mechanical resistance $\left(R_{m}\right)$. The 


\section{J. L. Tiernego and J. J. van Dixhoorn}

electromechanical energy conversion is described by a gyrator (GY) having a ratio $K$. In many applications the inductance can be neglected. Furthermore Thévenin's Theorem can be used to change the voltage source to an equivalent current source. Finally the electrical components can be reduced on the mechanical side by eliminating the gyrator. The result is a simple drive model, which corresponds to a linear torque-speed characteristic for a linear $\boldsymbol{R}$, having $R=R_{m}+K^{2} / R_{a}$. The complete bond graph is shown in Fig. 7. The multiport MTFs of previous figures have been expanded in twoport MTFs. Integrators are added at the 1 -junction of $\dot{\alpha}$ and $\dot{\beta}$ to account for the state variables $\alpha$ and $\boldsymbol{\beta}$ modulating these MTFs. In case the model is used for control system studies, the state variables $\alpha, \beta$ and $\gamma$ are to be fed back in an appropriate way to the $S_{E}$ elements. To that end the latter function as controlled sources.

\section{System order and equations from the bond graph}

The bond graph is made causal (Fig. 7) to determine the number of necessary and sufficient state variables. It is found that only three $I$-elements can be chosen in integral causality, each contributing one state variable. The other four $I$-elements are dependent storage elements indicated by $D I$ and to be calculated in differential causality using the previously defined state variables. In the causal assignment procedure $I_{x 1}$ is found to be always an

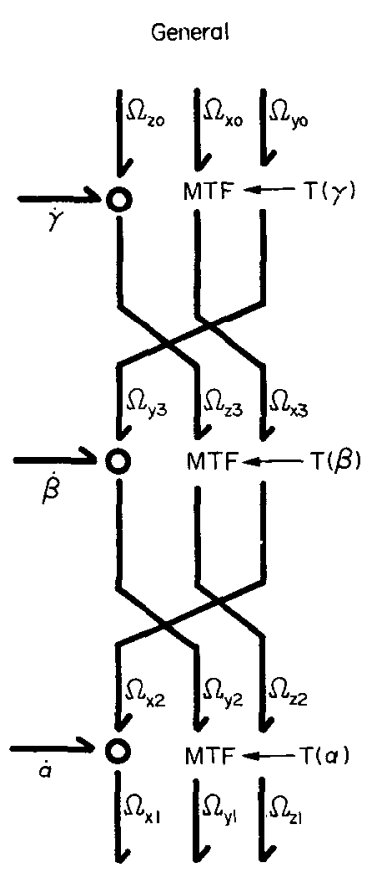

(a)

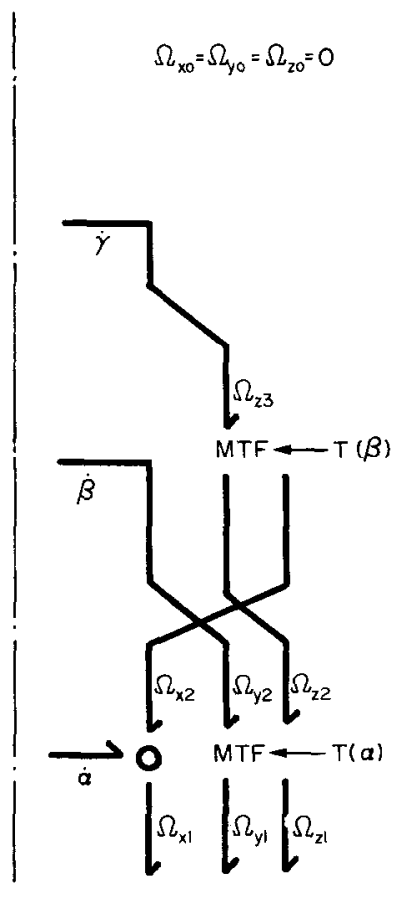

(b)

FIG. 4. Coordinate transformations in flight table geometry. 


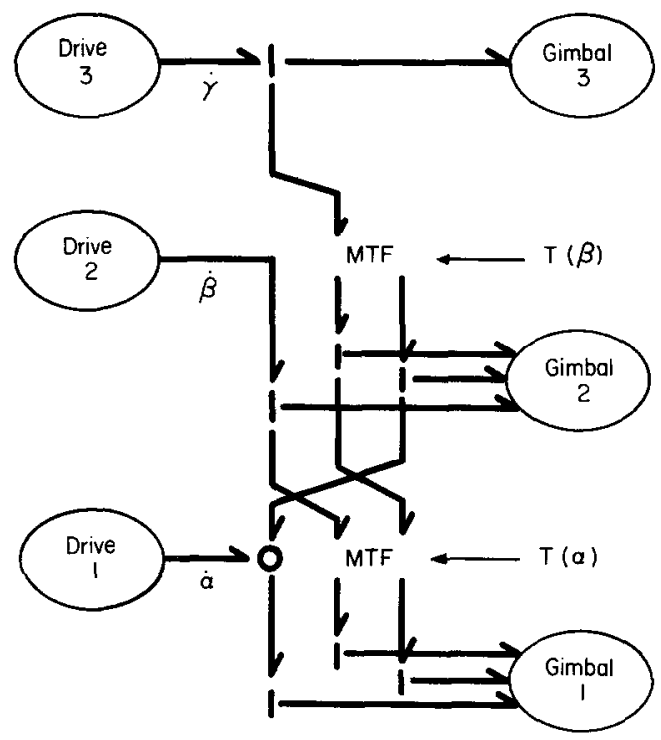

Fig. 5. Complete word bond graph.

independent storage element. The choice of the other two integral causality $I$-elements is arbitrary; an obvious choice has been made in Fig. 7.

The bond graph shows that, apart from the three velocity state variables from independent storage elements, two more state variables are required, because the MTFs are modulated by functions of displacements $\alpha$ and $\beta$. So a complete set of state variables can be: $\dot{\alpha}-\dot{\gamma} \mathrm{s} \beta, \dot{\beta}, \dot{\gamma}, \alpha$ and $\beta$. The dynamic system is of the fifth order only. It is interesting that $\gamma$ is not a state variable. Its initial condition does not influence the dynamics.

The output of the $\gamma$-integrator may be interesting as an observed variable, but is not connected to the system. In the linear case $\gamma$ just adds a zero
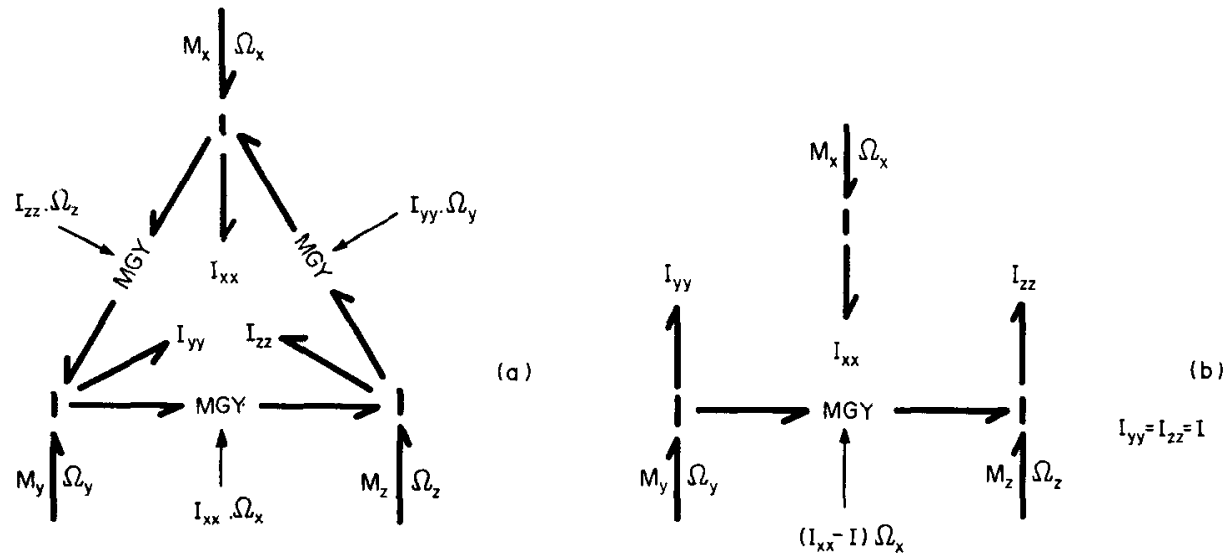

Fig. 6. Dynamics of a rotating body. 
eigenvalue. In the controlled system $\gamma$ is of course required and the system becomes of the sixth order.

If it is desired to have equations available, they can easily be written from the bond graph ( $\mathrm{s}$ and $\mathrm{c}$ are used for sin and cos)

$$
\begin{aligned}
& I_{x 1} \frac{\mathrm{d}(\dot{\alpha}-\dot{\gamma} \mathrm{s} \beta)}{\mathrm{d} t}=M_{\alpha}+\left(I_{y 1}-I_{z 1}\right) \Omega_{\mathrm{y} 1} \Omega_{z 1}, \\
& \begin{aligned}
I_{y 2} \cdot \frac{\mathrm{d} \dot{\beta}}{\mathrm{d} t}= & M_{\beta}+\left(I_{z 2}-I_{x 2}\right) \Omega_{z 2} \Omega_{x 2}+s \alpha \cdot I_{z 1} \cdot \dot{\Omega}_{z 1} \\
& +s \alpha\left(I_{y 1}-I_{x 1}\right) \Omega_{x 1} \Omega_{y 1}-c \alpha \cdot I_{y 1} \cdot \dot{\Omega}_{y 1}
\end{aligned} \\
& -c \alpha\left(I_{x 1}-I_{z 1}\right) \Omega_{x 1} \Omega_{z 1} \text {, } \\
& I_{z 3} \cdot \frac{\mathrm{d} \dot{\gamma}}{\mathrm{d} t}=M_{\gamma}+\mathrm{s} \beta I_{x 2} \dot{\Omega}_{x 2}+\mathrm{s} \beta M_{\alpha}+\mathrm{s} \beta\left(I_{z 2}-I_{y 2}\right) \Omega_{z 2} \Omega_{y 2} \\
& -c \beta\left(I_{y 2}-I_{x 2}\right) \Omega_{y 2} \Omega_{x 2}-c \beta s \alpha I_{y 1} \dot{\Omega}_{y 1} \\
& -c \beta s \alpha\left(I_{x 1}-I_{z 1}\right) \Omega_{x 1} \Omega_{z 1}-c \beta s \alpha I_{z 1} \dot{\Omega}_{z 1} \\
& -\mathrm{c} \beta \mathrm{s} \alpha\left(I_{y 1}-I_{x 1}\right) \Omega_{y 1} \Omega_{x 1}, \\
& \frac{\mathrm{d} \alpha}{\mathrm{d} t}=\dot{\alpha}, \\
& \frac{\mathrm{d} \boldsymbol{\beta}}{\mathrm{d} t}=\dot{\boldsymbol{\beta}} \text {. }
\end{aligned}
$$

As could be expected, the presence of differentiating DI-elements, producing $\dot{\Omega}$-terms to the right of the equal sign, results in a partly implicit system of non-linear state equations. Some tricks to solve this system by numerical integration are treated in the next section.

The system can also be rearranged to full differential causality. By putting exclusively $M_{\alpha}, M_{\beta}$ and $M_{\gamma}$ to the left of the equal sign and by expressing all angular velocities in $\dot{\alpha}, \dot{\beta}, \dot{\gamma}, \alpha$ and $\beta$, the equations become identical to those found by the Lagrangian approach. A method to solve such equations in complete differential causality is treated in the section on Lagrangian equations.

\section{Digital Simulation of the Bond Graph}

A major advantage of the bond graph approach is that the tedious writing of equations can normally be avoided. If no elements in differential causality exist the structure as it is can be used as an input to a mixed bond graph-block diagram simulation language like THTSIM (2). It takes some more work to use CSMP or any other package to solve a system of differential equations. For a small linear system ENPORT (3) simply accepts the bond graph directly and solves it by the transition matrix method. 
If some elements in differential causality exist, the loops in which those occur can be found in the causal bond graph. Some local remedies can often be introduced and the resulting bond graph can be simulated as described before. This will be the subject of the next section.

If in a part of a system many elements in differential causality exist and if they arc strongly coupled, as it happens in kinematic constructions, special procedures are required (4).

For linear systems the foregoing difficulties do not arise. ENPORT fi. treats such bond graphs without difficulty. Differential causality will result in a system of the form

$$
\mathbf{E} \dot{\mathbf{x}}=\mathbf{A x}+\mathbf{B u} .
$$

After left-multiplication with $\mathbf{E}^{-1}$ the system is solved in the standard way.

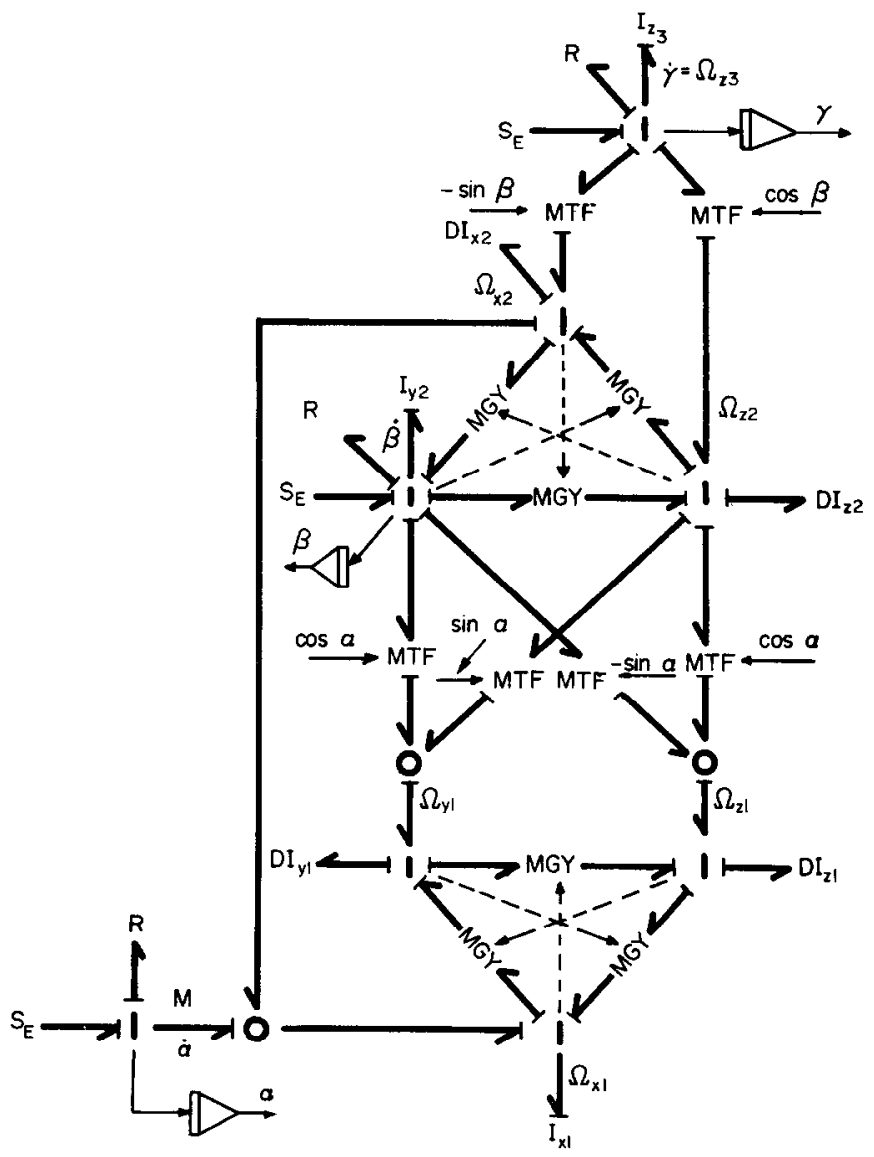

Fig. 7. Complete bond graph of the table. 
Dependent storage elements separated by a modulated transformer

In Fig. 8(a) this often occurring causal conflict is depicted in an elementary form. The MTF has a variable transformation ratio

$$
n(t)=\frac{e_{1}}{e_{2}} .
$$

To solve the computational problem two solutions exist

(1) delayed differentiation (derivative causality) of the smallest I-element;

(2) transformation of one $I$-element to the other side of the MTF.

Differentiation of $I_{2}$ (or $I_{1}$ ) is shown in Fig. 8(b) [or 8(c)]. In both cases a feedback loop occurs. This can readily be seen as the causal arrows between the the elements point in one direction and there are no gyrators.

If a differentiation procedure would exist that could cancel integration, these loops would be algebraic, having the indicated gains. A realisable differentiation procedure however incorporates a delay of at least one time step. Feedback loops with a delay are generally stable if the loop gain is less than 1 . So in a practical case there is only one stable choice between Figs. 8(b) and 8(c). The choice implies that the $I$-element, having the smallest effort (torque) at the same 1 -junction, has to be in differential causality.

The method works well if the loop gain is much less than 1 , which is clear, because the $D I$-element then is essentially negligible. If the loop gain approximates 1 , large, slowly damped errors occur at sudden transients. The method breaks down completely, if changes in the variable transformation ratio can make the loop gain greater than 1 . The implementation of delayed differentiation is easy; in THTSIM even special DI and DC blocks are available. The area of application however is restricted and needs careful analysis beforehand. Moreover the results are always approximations.

Transformation to the other side of the MTF is shown in Fig. 9(a). Here $I_{2}$ is transformed to the primary and can be combined with $I_{1}$ to form one element

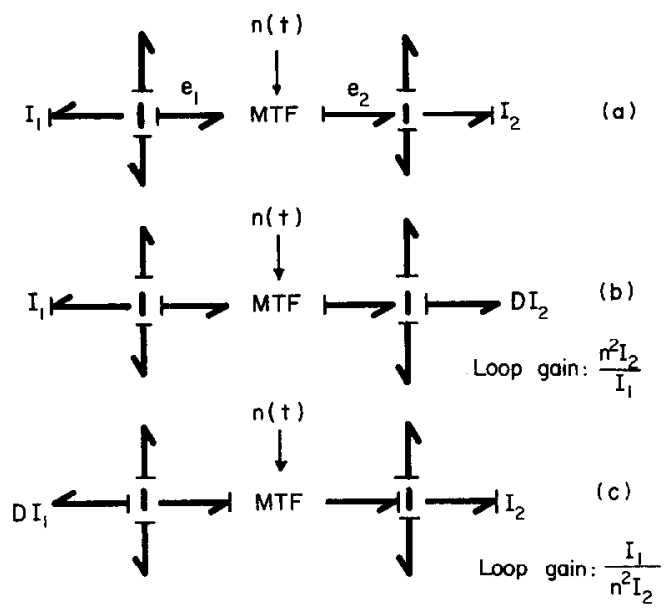

FIG. 8. Dependent storage elements solved by differentiation. 


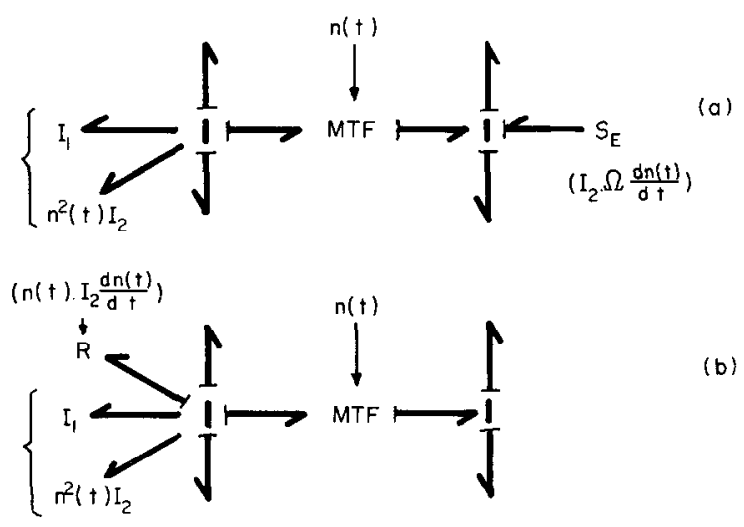

Fig. 9. Transformation of dependent inertia $I_{2}$.

$I_{1}+n^{2}(t) I_{2}$. At the original place of $I_{2}$ a controlled effort source $S_{E}=$ $I_{2} \cdot \Omega \cdot \mathrm{d} n / \mathrm{d} t$ with the indicated orientation has to be added. It will be clear that neither $n^{2} I_{2}$ nor $S_{E}$ do represent physical entities. A storage element whose energy content can be varied by a powerless signal violates energy conservation. The combination of $n^{2} I_{2}$ and $S_{E}$ however is physically sound. Also the torques to the left and right of the MTF are not measurable in the real system, however the velocities are. The method will, in general, not require an approximate differentiation. The modulating signal $n(t)$ of the MTF is often a function of displacement, which implies that $\mathrm{d} n / \mathrm{d} t$ is a function of a velocity of the system.

It is also possible to transform the $S_{\mathrm{E}}$ source to the left side of the MTF [Fig. 9(b)]. The transformed $S_{E}$ source will have the value $n(t) \cdot I_{2} \cdot \mathrm{d} n / \mathrm{d} t \cdot \Omega$. This $S_{E}$ is controlled by the velocity of the 1 -junction which it acts on. It can be replaced by a kind of resistor with value $n(t) I_{2} \mathrm{~d} n / \mathrm{d} t$, called a gyristor by Allen (9).

\section{Dependent Inertia-Elements in the Flight Table}

In assigning causality to the bond graph of Fig. 7 the choices have been made to take $I_{z 3}$ and $I_{\mathrm{y} 2}$ in integral causality. These choices result in a number of feedback loops starting at $I_{z 3}$ and $I_{\mathrm{y} 2}$ and incorporating the $D I$-elements, as indicated in Figs. 10(a) and (b). Also loops starting at $D I_{z 1}$ and $D I_{y 1}$ exist. As noticed before, a feedback loop can be found by following causal arrows pointing in one direction. The gain of the loop depends on the squared MTF ratio. Bearing this in mind it can be noticed immediately that all loop gains in Figs. 10(a) and 10(b) will have squares of sine and cosine functions in the numerator. Every other causality would bring a trigonometric function to the denominator and result in the possibility of an infinite loop gain. 
In Fig. 10(a) the $I_{y_{2}}$ element forms a part of two loops, whose gains have to be added. The total gain is

$$
\frac{\mathrm{s}^{2} \alpha I_{z 1}+\mathrm{c}^{2} \beta I_{\mathrm{y} 1}}{I_{\mathrm{y} 2}}
$$

In Fig. $10(\mathrm{~b})$ the total loop gain containing $I_{z 3}$ is

$$
\frac{\mathrm{s}^{2} \beta I_{x 2}+\mathrm{c}^{2} \beta\left(I_{z 2}+\mathrm{c}^{2} \alpha I_{z 1}+\mathrm{s}^{2} \alpha I_{\mathrm{y} 1}\right)}{I_{z 3}} .
$$

Taking into account the values of the inertia elements, the first loop gain is less than 0.5 . The second loop gain shows an area of values of $\alpha$ and $\beta$ in which the loop gain is greater than 1 . The loop gains starting at $D I_{z 1}$ and $D I_{y 1}$ can be evaluated in the same way. Both are much less than 1.

The loop gain analysis indicated that in the second loop, transformations of $I_{z 2}$ and $I_{x 2}$ to $I_{z 3}$ have to be applied. In the other loops differentiation of $I_{z 1}$ and $I_{y_{1}}$ can be sufficient. This part of the bond graph now changes, as illustrated in Fig. 11. If the simulation results had been unsatisfactory, which was not the case, $I_{z 1}$ and $I_{y 1}$ each could have been transformed to the other side of the two MTFs, a procedure which is more cumbersome than the simple one, but still feasible.

\section{Lagrangian Solution}

The Lagrangian solution is a standard approach to this kind of problem and will be treated in an abbreviated way. Apart from the classical method, leading to three non-linear second order equations, another and shorter method will be applied, leading to a more simple set of first order equations.

As in the bond graph approach, body coordinate systems have to be chosen, to be able to express the kinetic coenergies of a rotating body in a compact

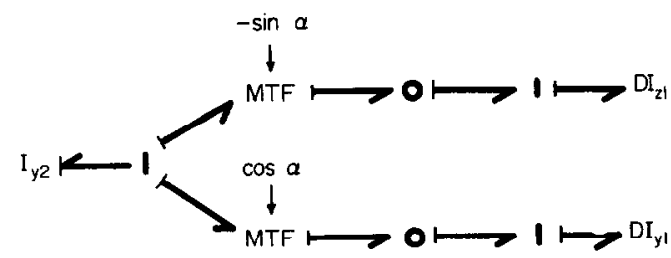

(a)

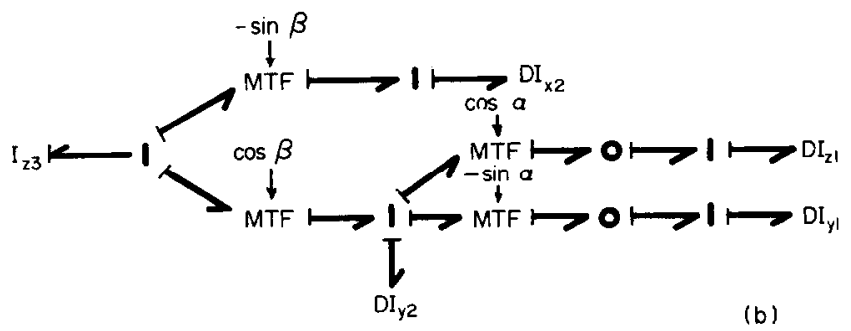

Frg. 10. Loops containing integration and differentiation. 


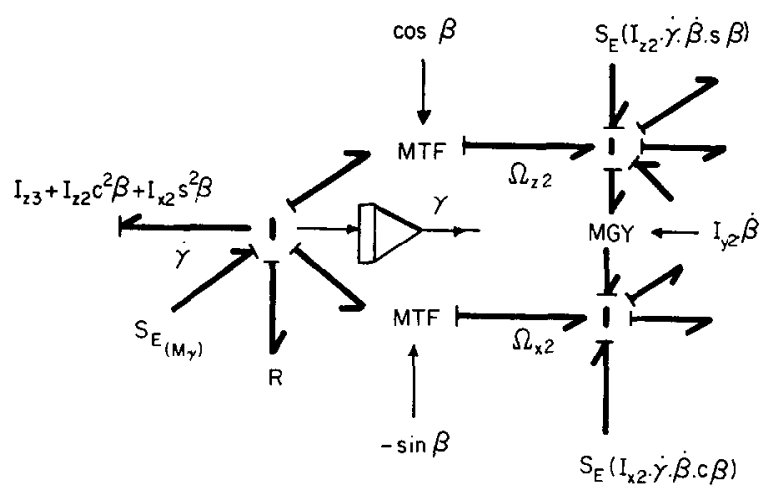

FIG. 11. Upper part of complete bond graph suitable for computation.

way. For the three gimbals the total kinetic coenergy $T^{*}$ is $2 T^{*}=I_{x 1} \cdot \Omega_{z 1}^{2}+I_{y 1} \cdot \Omega_{y 1}^{2}+I_{z 1} \cdot \Omega_{z 1}^{2}+I_{x 2} \cdot \Omega_{x 2}^{2}+I_{y 2} \cdot \Omega_{y 2}^{2}+I_{z} 2 \cdot \Omega_{z 2}^{2}+I_{z} 3 \cdot \Omega_{z 3}^{2}$.

The choice of the generalised coordinates is obviously $\alpha, \beta$ and $\gamma$. Again, as in the bond graph approach, the relations between these variables and the body angular velocities have to be established, giving

$$
\begin{aligned}
& \Omega_{x 1}=\dot{\alpha}-\mathrm{s} \beta \dot{\gamma}, \quad \Omega_{x 2}=-\mathrm{s} \beta \dot{\gamma}, \quad \Omega_{x 3}=0, \\
& \Omega_{y 1}=\operatorname{c} \alpha \dot{\beta}+\operatorname{s} \alpha c \beta \dot{\gamma}, \quad \Omega_{y 2}=\dot{\beta}, \quad \Omega_{y 3}=0 \text {, } \\
& \Omega_{z 1}=-s \alpha \dot{\beta}+c \alpha c \beta \dot{\gamma}, \quad \Omega_{z 2}=c \beta \dot{\gamma}, \quad \Omega_{z 3}=\dot{\gamma} .
\end{aligned}
$$

Next $T^{*}$ is expressed in $\dot{\alpha}, \dot{\beta}, \dot{\gamma}, \alpha$ and $\beta$.

Because no potential energy storage elements exist the Lagrangian $L=T^{*}$. The Lagrangian equations, resulting in the generalised forces (torques $M$ ) reduce to

$$
M_{x}=\frac{\mathrm{d}\left(\partial T^{*} / \partial \dot{x}\right)}{\mathrm{d} t}-\frac{\partial T^{*}}{\partial x}, \quad(x=\alpha, \beta, \gamma)
$$

After elaborate evaluation of these equations $M_{\alpha}, M_{\beta}$ and $M_{\gamma}$ are found and can be written in matrix notation

$$
\left[\begin{array}{c}
M_{\alpha} \\
M_{\beta} \\
M_{\gamma}
\end{array}\right]=\mathbf{A}\left[\begin{array}{c}
\dot{\alpha} \\
\ddot{\beta} \\
\ddot{\gamma}
\end{array}\right]+\mathbf{B}\left[\begin{array}{c}
\dot{\alpha}^{2} \\
\dot{\beta}^{2} \\
\dot{\gamma}^{2}
\end{array}\right]+\mathbf{C}\left[\begin{array}{cc}
\dot{\alpha} & \dot{\beta} \\
\dot{\beta} & \dot{\gamma} \\
\dot{\gamma} & \dot{\alpha}
\end{array}\right] .
$$

These equations correspond with those previously derived from the bond graph. As is always the case using the Lagrangian method, the equations are in derivative causality and not easy to solve because the torques are given and the velocities and displacements have to be found.

To bring the system into integral causality the $\mathbf{A}$-matrix has to be inverted. With some effort this can be done analytical. A brute force method is numerical inversion by the computer at every time step of the integration 


\section{J. L. Tiernego and J. J. van Dixhoorn}

routine. The scheme for the solution of the complete set of equations can now be found, as illustrated in Fig. 12(a).

An efficient alternative procedure which simplifies the analytical work has been proposed by Vance and Sitchin $(\mathbf{5 , 6 )}$. By definition of generalised momenta

$$
H_{x}=\frac{\partial T^{*}}{\partial x} \quad(x=\alpha, \beta, \gamma) .
$$

Lagranges equation can be written

$$
\dot{H}_{x}=\frac{\partial T^{*}}{\partial x}+M_{x} .
$$

The analytical differentiation of $\partial T^{*} / \partial \dot{x}$ with respect to time, which was most of the work in the previous formulation, does not have to be performed. For momenta it is found that

$$
\left[\begin{array}{c}
H_{\alpha} \\
H_{\beta} \\
H \gamma
\end{array}\right]=\mathbf{A}\left[\begin{array}{c}
\dot{\alpha} \\
\dot{\beta} \\
\dot{\gamma}
\end{array}\right] \text { or } \quad \mathbf{H}=\mathbf{A} \cdot \dot{\mathbf{x}},
$$

in which the same $\mathbf{A}$ matrix occurs as before

$\mathbf{A}=\left[\begin{array}{c:c}I_{x 1} & 0 \\ \hdashline 0 & I_{y 2}+I_{y y} c^{2} \alpha+I_{z 1} \mathrm{~s}^{2} \alpha \\ \hdashline-I_{x 1} \cdot \mathrm{s} \beta & \left(I_{y 1}-I_{z 1}\right) \mathrm{s} \alpha \cdot \mathrm{c} \alpha \cdot \mathrm{c} \beta\end{array}\right.$

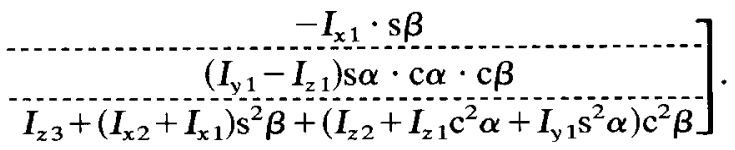

For $\dot{H}_{x}$ it is found that

$$
\dot{\mathbf{H}}=\mathbf{B}_{1}\left[\begin{array}{c}
\dot{\alpha}^{2} \\
\dot{\boldsymbol{\beta}}^{2} \\
\dot{\gamma}^{2}
\end{array}\right]+\mathbf{C}_{1}\left[\begin{array}{c}
\dot{\alpha} \dot{\beta} \\
\dot{\boldsymbol{\beta}} \dot{\gamma} \\
\dot{\gamma} \dot{\alpha}
\end{array}\right]+\mathbf{M}
$$

in which

$$
\begin{gathered}
\mathbf{B}_{1}=\left[\begin{array}{c:c:c}
0 & -\left(I_{y 1}-I_{z 1}\right) \mathrm{s} \alpha \cdot \mathrm{c} \alpha & \left(I_{I_{1}}-I_{z 1}\right) \mathrm{s} \alpha \mathrm{c} \alpha \mathrm{c}^{2} \beta \\
\hdashline 0 & 0 & \left(I_{x 2}+I_{x 1}-I_{z 2}+I_{z 1}-\left(I_{y 1}-I_{21}\right) \mathrm{s} \alpha\right) \mathrm{s} \beta \mathrm{c} \beta \\
\hdashline 0 & 0 & 0
\end{array}\right], \\
\mathbf{C}_{1}=\left[\begin{array}{ccc}
0 & \left(I_{y 1}-I_{z 1}\right)\left(\mathrm{c}^{2} \alpha-\mathrm{s}^{2} \alpha\right) \mathrm{c} \beta & 0 \\
0 & -\left(I_{y 1}-I_{z 1}\right) \mathrm{s} \alpha \mathrm{c} \alpha \mathrm{s} \beta & -I_{x 1} \mathrm{c} \beta \\
0 & 0 & 0
\end{array}\right] .
\end{gathered}
$$




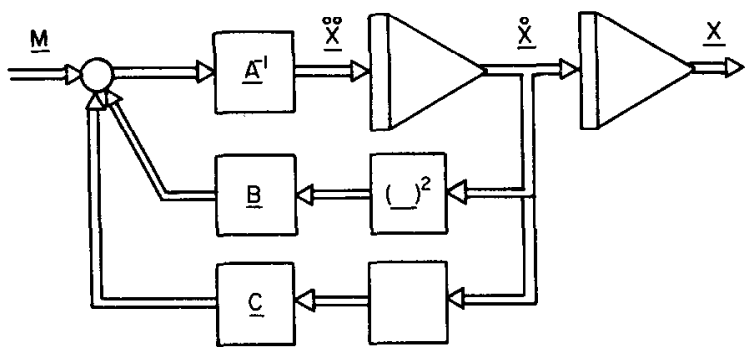

(a)

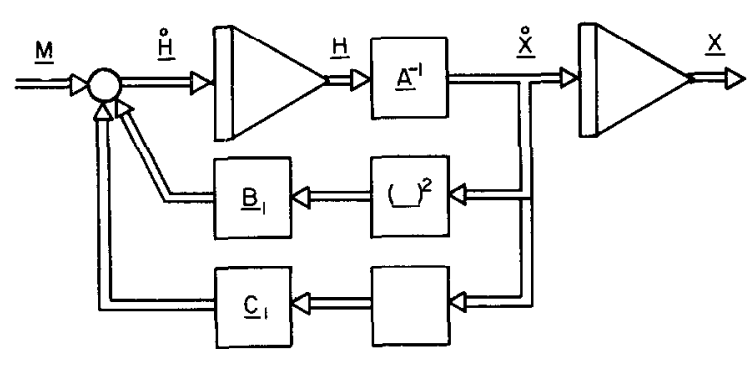

(b)

Frg. 12. Schematic solution of the Lagrangian equations.

The solution of this set of equations is shown in Fig. 12(b). As in the first method the A-matrix has to be inverted. The advantages of the second method, however, are that the computer solution is quicker because the $\mathbf{B}_{1}$ and $\mathbf{C}_{1}$ matrices contain less terms than the $\mathbf{B}$ and $\mathbf{C}$ matrices, as well as that the analytical work in deriving the $\mathbf{A}, \mathbf{B}_{1}$ and $\mathbf{C}_{1}$ matrices is much less.

\section{Comparison of Bond Graph and Lagrangian Methods}

It has already been shown that both methods lead to the same equations, which proves the formal validity of the bond graph. To compare the numerical solubility both methods were digitally simulated using THTSIM, incorporating a fixed interval second order Adams-Bashfort integration. In the bond graph the dependent inertias $I_{z 1}$ and $I_{y 1}$ were approximated by delayed differentiation; $I_{x 2}$ and $I_{z 2}$ were transformed to $I_{z 3}$. For the Lagrangian solution the second formulation method was chosen using analytical inversion of the A-matrix. Rectangular test pulses were applied in $M_{\alpha}, M_{\beta}$ and $M_{\gamma}$. Some of the simulation results are shown in Fig. 13(a) and 13(b). In these figures the pulse was given at $M_{\gamma}$. That pulse produced the largest difference between the Lagrangian and bond graph solution. Figures 13(a) and 13(b) show the angles $\gamma$ and $\beta$ computed from the I agrangian equations as well as the difference $\Delta \gamma$ and $\Delta \beta$ between both solution methods, for three different values of the integration time step. Both methods require a time step less than $0.01 \mathrm{~s}$ to produce a 
M. J. L. Tiernego and J. J. van Dixhoorn

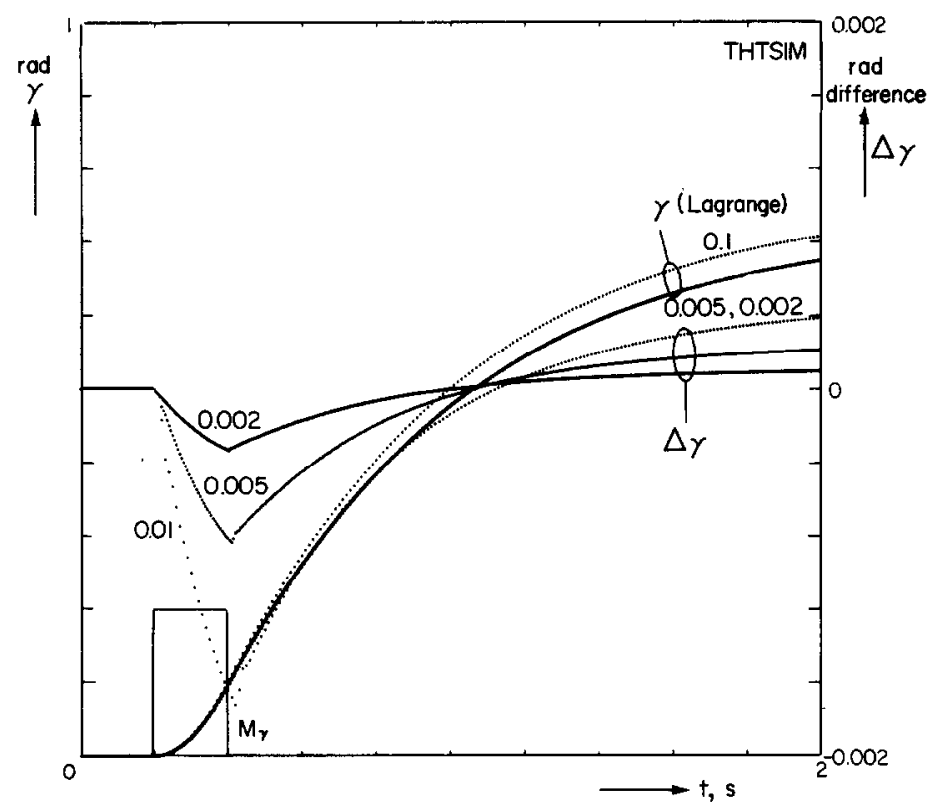

Fig. 13(a). Difference between Lagrangian and bond graph simulation of $\gamma$.

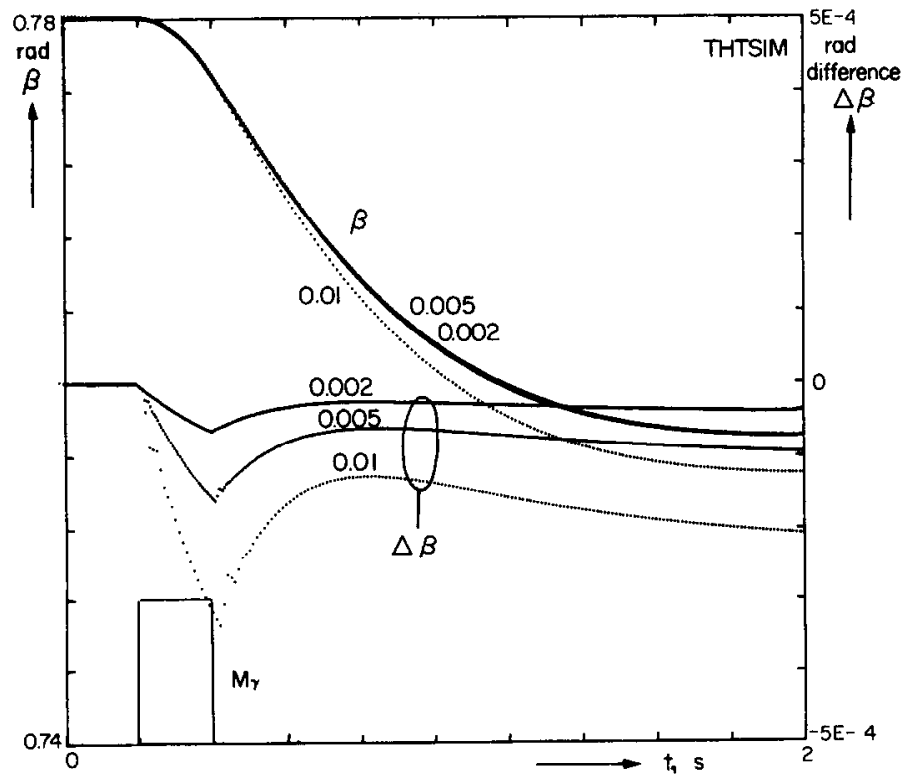

FIG. 13(b). Difference between Lagrangian and bond graph simulation of $\beta$. 


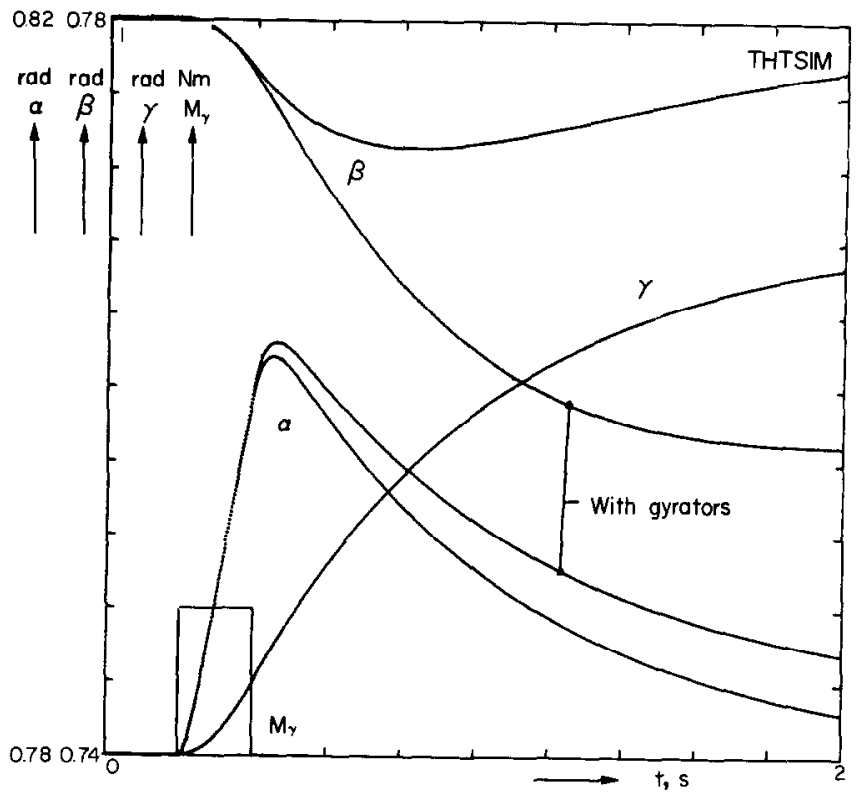

FIG. 13(c). Simulation result of the bond graph with the MGYs omitted compared with the complete bond graph.

trajectory which is roughly independent of the time step. This is comprehensible for this open loop double integrating system. For a time step of $0.002 \mathrm{~s}$ the ultimate difference between both methods is shown to be less than $5 \times 10^{-5} \mathrm{rad}$, which can be considered very small. The largest differences occur at the transients of the applied pulse as could be expected due to the differentiating elements. As to the computing time of the Lagrangian and bond graph method can be said that the Lagrangian solution time was with the same delta about $3 \%$ longer.

The bond graph can be used to determine what the effects of simplification of the model are. Figure 13(c) shows a comparison between the complete model and the model with the MGYs omitted. A strongly different transient and static behaviour resulted.

A strong point in favour of the bond graph model is, that all physical variables are explicitly available, so that their values can easily be plotted. More important, however, is that the model can easily be extended with new components, like springs, dampers, Coulomb friction etc. Such components can generally be attached or eliminated without upsetting the structure of the model. Using an interactive language like THTSIM, the effect can immediately be seen on the display.

An unexpected advantage of the bond graph method over the Lagrangian approach was discovered during the first simulation runs, when the models still gave erroneous results. The transparancy of the bond graph and the availability of all variables made error localisation relatively easy. Errors in the Lagrangian model in contrast required a check of all equations. 


\section{Simplified and More Complex Cases}

\section{Simplified cases}

To avoid the complexity of the previous models in practical situations often simplified models are used, a tendency which is reflected in literature, e.g. (7). Many of the properties which can be used in such simplifications are, with some experience, visible in the bond graph. If the rotating body is completely symmetrical $\left(I_{x x}=I_{y y}=I_{z z}\right)$ the complete MGY-ring structure disappears. If the body is partly symmetrical, so as to have two equal moments of inertia, the coupling with the third one disappears, as is indicated in Fig. 6(b). At low angular velocities the gyration ratios of the MGY-components become very small and the MGY-ring can be considered to disappear partly or completely.

Asymmetrical and unbalanced table

In the foregoing, the bond graph and Lagrangian models have been compared for the case of a symmetrical and balanced flight table. For sake of completeness it will be shown that the bond graph can be extended to more general cases. Computation of both kinds of models requires much more effort and will not be treated here.

An asymmetrical table occurs when the physical rotational axes do not coincide with the principal axes of the three gimbals. It is assumed that if the body coordinate system is chosen along the rotational axes the centres of mass and the origin still coincide. In this case the simple inertia structure of the gimbal dynamics, as shown in Fig. 6, has to be extended. The inertia matrix is not diagonal any more; the three separate $I$-components have to be replaced by a three-port $I$-field (1). Computational causality will require the $I$-matrix to be manipulated into a partly inverted form.

An unbalanced table occurs when the centre of mass of a gimbal does not coincide with the origin of the rotational and coordinate axes. This happens for instance when a mass is placed on the table. Translational velocities and forces, including gravity, have to be taken into account. Suppose the unbalance mass is located at the coordinates $\left(x_{1}, y_{1}, z_{1}\right)$ of the body coordinate system. The coupling between translational velocities $\left(\dot{x}_{1}, \dot{y}_{1}, \dot{z}_{1}\right)$, expressed in the body coordinate system and the rotation of this system can be described by a multiport transformer having the following transformation matrix $\operatorname{MTF}\left(x_{1}, y_{1}, z_{1}\right)$ :

$$
\left[\begin{array}{l}
\dot{x}_{1} \\
\dot{y}_{1} \\
\dot{z}_{1}
\end{array}\right]=\left[\begin{array}{ccc}
0 & z_{1} & -y_{1} \\
-z_{1} & 0 & x_{1} \\
y_{1} & -x_{1} & 0
\end{array}\right]\left[\begin{array}{l}
\Omega_{x} \\
\Omega_{y} \\
\Omega_{z}
\end{array}\right]
$$

The inertial properties of the unbalance mass $(m)$ and the gravity force $(m g)$ have their most simple form in the inertial coordinate system. The transformation matrix $\operatorname{MTF}(\alpha, \beta, \gamma)$ to the velocities $\left(\dot{x}_{1,0}, \dot{y}_{1,0}, \dot{z}_{1,0}\right)$ in the incrtial system is

$$
\left[\begin{array}{l}
\dot{x}_{1,0} \\
\dot{y}_{1,0} \\
\dot{z}_{1,0}
\end{array}\right]=\left[\begin{array}{ccc}
c \beta \cdot c \gamma & -c \alpha \cdot s \gamma+s \alpha \cdot s \beta \cdot c \gamma & s \alpha \cdot s \gamma+c \alpha \cdot s \beta \cdot c \gamma \\
c \beta \cdot s \gamma & c \alpha \cdot c \gamma+s \alpha \cdot s \beta \cdot s \gamma & -s \alpha \cdot c \gamma+c \alpha \cdot s \beta \cdot s \gamma \\
-s \beta & s \alpha \cdot c \beta & c \alpha \cdot c \beta
\end{array}\right]\left[\begin{array}{l}
\dot{x}_{1} \\
\dot{y}_{1} \\
\dot{z}_{1}
\end{array}\right]
$$




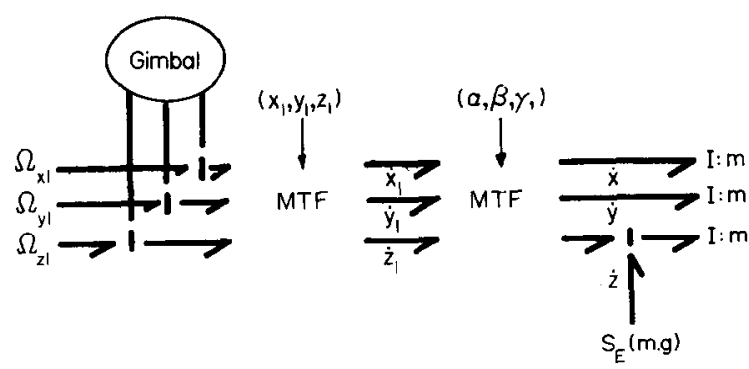

FIG. 14. Extended bond graph representing mass unbalance at gimbal 1.

The bond graph of the gimbal dynamics can now be extended with the unbalance mass $(m)$ and its associated gravity force, as indicated in Fig. 14.

If this bond graph would be used for computation, the three $m$-components, being dependent masses, would have to be taken in derivative causality. To avoid this, it is advantageous to transform the three $m$ 's to the left side of both MTFs and add them to the inertia matrix of the gimbal. The result is in accordance with the Huygens-Steiner rule

$$
\begin{array}{ll}
I_{x x}^{\prime}=I_{x x}+m\left(y_{1}^{2}+z_{1}^{2}\right), & I_{x y}^{\prime}=I_{x y}+m \cdot x_{1} y_{1}, \\
I_{y y}^{\prime}=I_{y y}+m\left(x_{1}^{2}+z_{1}^{2}\right), & I_{x z}^{\prime}=I_{x z}+m \cdot x_{1} z_{1}, \\
I_{z z}^{\prime}=I_{z z}+m\left(x_{1}^{2}+y_{1}^{2}\right), & I_{y z}^{\prime}=I_{y z}+m \cdot y_{1} z_{1} .
\end{array}
$$

\section{Conclusions}

It has been shown how, by a three-fold application of a planar coordinate transformation, a bond graph of the geometry of a flight table can be derived. The inertial properties of the gimbals can be added in a standard way. The resulting bond graph clearly shows the physical structure of the system. The equations of motion can be derived from the bond graph and agree with those derived by the Lagrangian approach.

The major computational problem is due to the many dependent inertia elements, being coupled in a non-linear way. Their presence naturally leads to a formulation of the equations in derivative causality, i.e. torques expressed as derivatives of velocitics. Their computation howcver requires an analytical or repeated numerical matrix inversion. In the Lagrangian approach this can never be avoided. In the bond graph of the table under consideration however it proved possible to apply local remedies to the coupled dependent inertias. So matrix inversion could be avoided and standard solution by numerical integration, using the interactive THTSIM bond graph and block diagram language was possible. One advantage is that all physical variables are at hand in the simulation model.

Moreover, components can be directly added to the model without changing the structure; in the Lagrangian approach the equations have to be derived all over again. The same advantage applies during the trouble shooting phase of 


\section{J. L. Tiernego and J. J. van Dixhoorn}

the simulation. For more complex platforms, showing intricate dependencies between inertias, the computational advantage of the bond graph will no longer hold and matrix inversion will be needed. The clarity of the model however still stands.

\section{References}

(1) D. Karnopp and R. C. Rosenberg, "Analysis and Simulation of Multiport Systems". M.I.T. Press, Cambridge (Mass), U.S.A., 1968.

(2) J. J. van Dixhoorn, "Simulation of bond graphs on minicomputers", Trans ASME, J. Dyn. Syst., Meas., Control, Vol. 99, No. 1, pp. 9-14, 1977.

(3) R. C. Rosenberg, "A User's Guide to Enport-4". Wiley-Interscience, New York, 1974.

(4) R. R. Allen and S. Dubowsky, "Mechanisms as components of dynamic systems; a bond graph approach", J. Engrg Ind, Trans ASME B, Vol. 99, No. 1, pp. 104-111, 1977.

(5) T. M. Vance and A. Sitchin, "Derivation of first-order difference equations for dynamic systems by direct application of Hamilton's principle", Trans ASME, $J$. Appl. Mech., Vol. 37, pp. 276-278, June 1970.

(6) D. Karnopp, "The energetic structure of multibody dynamic systems", in "System Structures in Engineering" (Edited by O. Bjørke and O. I. Franksen), TAPIR Trondheim, Norway, 1978.

(7) J. Weislitzer and U. Bloch, "A three axis flight table with dc torque motors", IEEE Trans., Vol. IECI-18, No. 4, pp. 117-133, Nov. 1971.

(8) D. Karnopp, "Lagranges equations for complex bond graph systems", Trans ASME, J. Dyn. Syst., Meas., Control, Vol. 99, No. 4, pp. 300-306, 1977.

(9) R. R. Allen, "Multiport representation of inertia properties of kinematic mechanisms", J. Franklin Inst., Vol. 308, pp. 235-253, 1979. 